\title{
Renal Calculi Detection in Ultrasound images and Diagnosis of Images using Image Segmentation
}

\author{
Mr. A. Irsath Ahamed, M.Sc., M.Phil., , Prof. B. Mohamed Faize Basha, MCA., \\ M.Phil., ${ }^{2}$ Prof. M. Mohamed Surputheen, M.Sc.,P.G.D.C.A.,M.Phil., ${ }^{3}$ \\ 1(Department of Computer Science, Jamal Mohamed College, India.) \\ 2(Department of Computer Science, Jamal Mohamed College, India.) \\ 3(Department of Computer Science, Jamal Mohamed College, India.)
}

\begin{abstract}
Now-a-days Renal Calculi is becoming a most common disease in both men and women. Calculi are due to abnormal collection of certain chemicals like oxalate, phosphate and uric acid. These calculi can be present in kidney, urethra or in urinary bladder. Most of the previous study in diagnosis of Renal Calculi spots out the presence or absence of the calculi in the kidney. In this paper we propose an algorithm to detect the renal calculi and to find the level of the calculi. It is more helpful to change the diet conditions. In this paper, we presented a method for ultrasound kidney image diagnosis for calculi and to find the size of the calculi using Advanced seeded region growing based segmentation. With segmented portions of the images the intensity threshold values helps to classify the level of the calculi as early calculi, medium calculi and large calculi. The Advanced Seeded Region Growing based image segmentation process homogeneous region depends on the image features. First, the regions of interest (ROIs) extracted from the pre-processed image. Second, the initial seed is selected based on ROIs extracted from the image. The size of the growing regions depends on this look up table entries. After completing region growing, region merging is used to suppress the high frequency artifacts. The diagnosis process is done based on the intensity threshold variation obtained from the segmented portions of the image and level of the calculi is found out.
\end{abstract}

Keywords: Renal Calculi, Advanced seeded region growing, intensity threshold, region merging, segmentation

\section{Introduction}

Renal calculi are becoming a most common problem, because of the living nature of people. ${ }^{[1]}$ The occurrence of renal stone is usually believed to be due to crystallisation of minerals inside urine, which act as the nidus for more sedimentation and finally the formation of a stone within the kidney. Calculi are due to abnormal collection of certain chemicals like oxalate, phosphate and uric acid. These calculi can be present in kidney, urethra or in urinary bladder. Most of the previous study in diagnosis of Renal Calculi spots out the presence or absence of the calculi in the kidney. In this paper we propose an algorithm to detect the renal calculi and to find the size of the calculi. It is more helpful to change the diet conditions. In this paper, we presented a method for ultrasound kidney image diagnosis for calculi and to find the size of the calculi using Advanced seeded region growing based segmentation.

\section{Ultrasound images:}

${ }^{[2]}$ In various imagining modalities, Ultrasound image is non-invasive, low cost imaging and flexible. Ultrasound image has more features like minimal scan time, flexible operation and reduced exposure to harmful radiation.

\section{Ultrasound Image Segmentation}

Advanced seeded Region Growing algorithm consist of following three steps, they are Seed selection, Region growing and merging and threshold value. ${ }^{[3]} \mathrm{A}$ seed is most valuable point and it is selected through user interaction. Using Advanced Seeded Region Growing algorithm, the seed point can start to grow. After completing growing the seed point we should merge the pixels. Threshold value is determined to cover the related pixels. Mean ratio and variance of the pixels is used as the parameters for seed selection. Using these parameters we can easily find out that the processed pixel is within homogeneous region or not. We can define the shape and size of the homogeneous regions for smoothing by using the advanced region growing algorithm.

The main theme of the Advanced Region growing algorithm is to extract the homogeneous pixels from the ultrasound image. Region growing technique is better in noisy images, where borders are extremely difficult to detect such as ultrasound medical images. For region growing method homogeneity is an important property, which can be based on gray-level, shape, model. For region- based segmentation, the basic requirement is to satisfy the region similarity in the kidney image. 


\section{Several important issues about region growing:}

\section{The suitable selection of seed points is important.}

${ }^{[4]}$ The selection of seed points is depending on the users. For example, in a gray-level lightning image, we may want to segment the lightning from the background. Then probably, we can examine the histogram and choose the seed points from the highest range of it.

\section{More information of the image is better.}

Obviously, the connectivity or pixel adjacent information is helpful for us to determine the threshold and seed points.

3. The value, "minimum area threshold".

No region in region growing method result will be smaller than this threshold in the segmented image.

4. The value, "Similarity threshold value".

If the difference of pixel-value or the difference value of average gray level of a set of pixels less than

"Similarity threshold value", the regions will be considered as a same region.

The Proposed Advanced seeded region growing algorithm for ultrasonic kidney images:

- Choose a window sized $(2 \mathrm{k}+1) \times(2 \mathrm{k}+1)$ being centered at $(\mathrm{i}, \mathrm{j})$ for seed point

- Generate the look up table for local statistics for each pixel:

- Calculate the homogeneity

- Calculate the statistical similarity bound

- Implement region growing for every pixel:

- This function performs "region growing" in an image from a specified seed point $(\mathrm{x}, \mathrm{y})$

$\mathrm{J}=$ region growing $(\mathrm{I}, \mathrm{x}, \mathrm{y}, \mathrm{t})$

I : input image

$\mathrm{J}:$ logical output image of region

$\mathrm{x}, \mathrm{y}:$ the position of the seedpoint

$\mathrm{t}:$ maximum intensity distance

The region is iteratively grown by comparing all unallocated neighbouring pixels to the region. The difference between a pixel's intensity value and the region's mean is used as a measure of similarity. The pixel with the smallest difference measured this way is allocated to the respective region. This process stops when the intensity difference between region mean and new pixel become larger than a certain threshold (t).

- Implement region merging:

- Labeling the each region with a unique number

- Store the neighboring region information for every seed region

- Merge the neighboring region according to the merging criteria with the seed region

- Update the segmented image output

Image diagnosis:

${ }^{[2]}$ Image diagnosis is based on the texture of the segmented portion of the images compared to that of standard benchmarked kidney image texture values. The Ultrasound kidney image is $\mathrm{x}(\mathrm{i}, \mathrm{j})$ ' $\mathrm{N}$ ' is the total number of pixels in kidney region "R". The first order gray level statistical features Mean Pixel Rate (M), Average absolute Deviation Pixel Rate (AD), Variance Pixel Rate (V) are estimated as:

Mean Pix el Rate $=\frac{1}{N} \sum_{R_{i, j} \in R} x(i, j)$

Average absolute deviation $=\frac{\mathbf{1}}{\mathbf{N}} \sum_{i=1}^{n}\left|x_{i}-m(x)\right|$

var riance PixelRate $=\frac{1}{N-1} \sum_{i=1}^{n}\left(x_{i}-m(x)\right)$

Experimentation of kidney image segmentation and classification:

Ultrasound kidney images with normal and stones of various sized is obtained from the medical laboratory. The edges of the stones were clearly observed on the segmented portion of the images with classification indicate the size of the stone presented. The images with segmented classification find the mean 
and deviation values of intensities of the pixels in the image. The seed is the starting point of the region growth. The position of the seed must be inside the calculus in the kidney image. The segmented image is then a binary image representing the inside and the outside of the calculus on the kidney image. The similarity criteria of the region growing algorithm allows to differentiating the calculus from the rest of the image. Hence, it is possible to study with calculi of different colors and textures to ensure a proper functioning in most of the clinical scenario.

The figure 1 shows the detection of Renal Calculus. The manual initialization obtain good results on the validation ultrasound kidney image classification and diagnosis of the stone presence and early stages composed of images coming from different clinical situations. The stone size formed in normal patient is less than $2 \mathrm{~mm}$ however with size of $5 \mathrm{~mm}$ and above cause serious issue to the kidney. The size of the stones in the kidney between 3-4 $\mathrm{mm}$ is classified as early calculi which shown in figure 2 .

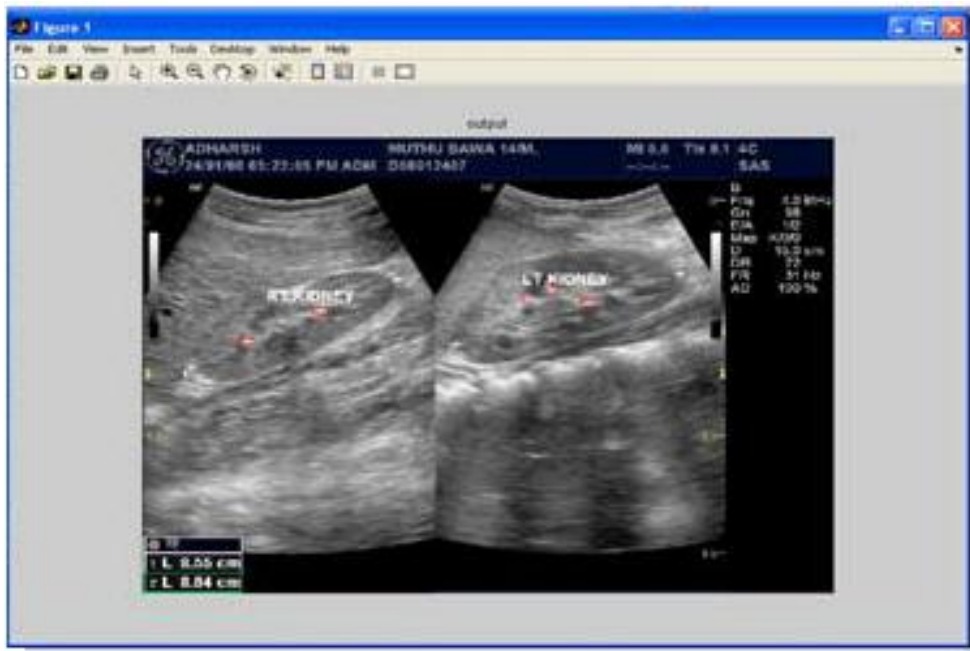

Figure 1 shows the detection of Renal Calculus

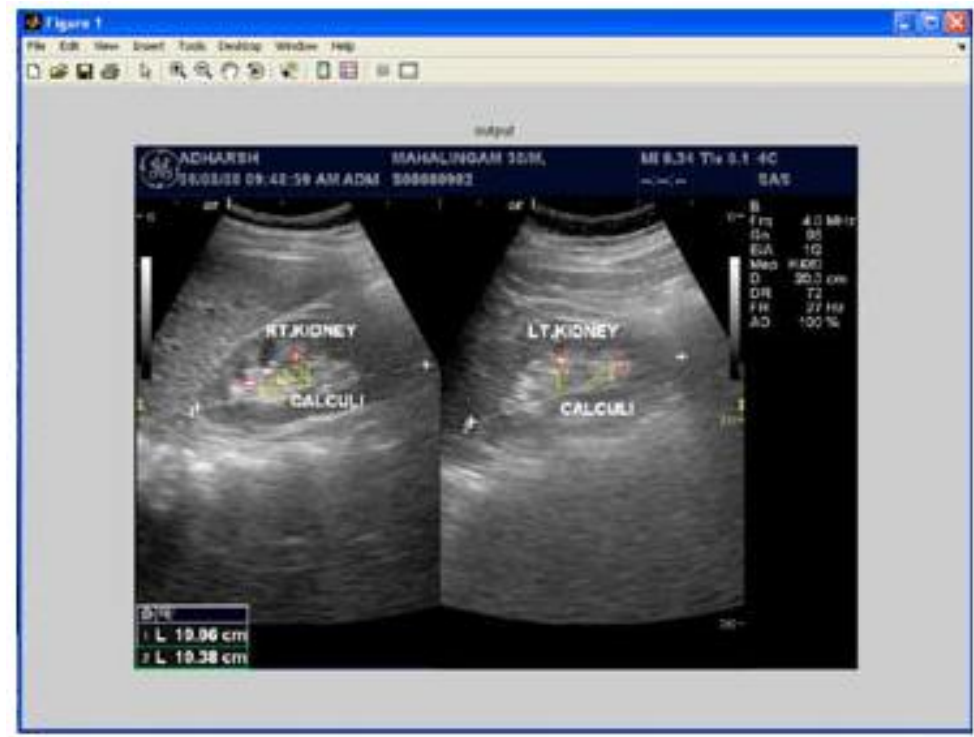

Figure 2 shows the early calculi

Table 1 shows the data obtained from Ultrasound image

\begin{tabular}{|c|c|c|c|}
\hline Type & Mean Pixel Rate & $\begin{array}{c}\text { Average absolute Deviation } \\
\text { Pixel Rate }\end{array}$ & Variance Pixel Rate \\
\hline Early Calculi & 22.1 & 6.1 & 2.3 \\
\hline Medium calculi & 25.3 & 8.5 & 4.1 \\
\hline Large Calculi & 27.1 & 11.3 & 7.3 \\
\hline
\end{tabular}


The extracted data from the Ultrasound image is tabulated above. The comparison of this data is plotted in figure3.

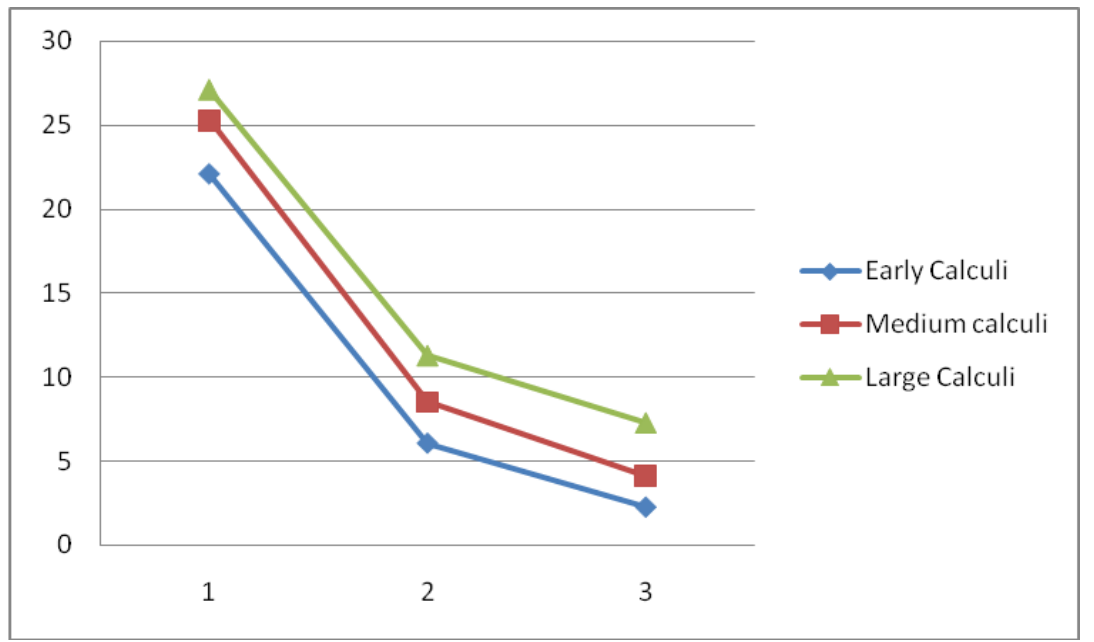

Figure 3 shows the comparison of data obtained from Ultrasound image

\section{Conclusion}

The study presented kidney stone detection and to find the level with integrated image segmentation and classification. The segmentation process is carried out with Advanced Seeded Region Growing method for identifying the intensity threshold variation. The experimentation is conducted to easy and effective diagnosis of stones and its early stages from ultrasound image samples taken from various patients. The parameters for diagnosing and classifying the US kidney stone images are identified and analyzed. The quality features describe the kidney stone size and its position is made viable. There has been significant difference observed in many parameters of each type of renal calculi. It is required to analyze different sizes of the kidney stone and based on these numerical values of features it is highly feasible to develop a universal reference for kidney stone categories.

\section{Reference}

[1]. Steve WH Chan, CF Ng CW Man, Robert Chung, SK Li, "A report on a randomly sampled questionnaire survey about renal stone disease in Hong Kong”, Hong Kong Med J 2008;14:427-31.

[2]. Ms.P.R. Tamilselvi, Dr.P. Thangaraj, "Computer Aided Diagnosis System for Stone Detection and Early Detection of Kidney Stones", Journal of Computer Science 7 (2): 250-254, 2011.

[3]. Dr. P. R. Tamiselvi, "Segmentation of Renal Calculi Using Squared Euclidean Distance Method", International Journal of Scientific Engineering and Technology, Volume No.2, Issue No.7, pp : 651-655.

[4]. Dr. P. R. Tamiselvi, "Effective Segmentation Approaches for Renal Calculi Segmentation", International Journal of Science and Research (IJSR), India Online ISSN: 2319-7064. 